\title{
An Epidemiologic Study on the Correlation between Clinical Symptomatology and Modality of Birth in Twins, 1990 to 1993
}

\author{
L. Cannatelli, E. Gitto, D. Prudente, V. Cordaro, S. Cordaro, C. Liotta, I. \\ Barberi
}

${ }^{1}$ Department of Paediatrics, University of Messina, Italy

\begin{abstract}
The neonatal intensive care of twins has seen many changes over later years. In fact, major improvements in prenatal evaluation and neonatal management techniques have greatly increased the possibility of survival for infants at risk, especially those born from multiple pregnancies. Our study reports the results of a retrospective survey for the years 1990 to 1993 , on the outcome of 76 twin infants (ie. $11,4 \%$ of the 661 newborns admitted to our unit over that period) correlating the clincial symptoms observed with the modality of birth.
\end{abstract}

Table

\begin{tabular}{lcccccc}
\hline & \multicolumn{3}{c}{ Spontaneous delivery } & \multicolumn{3}{c}{ Caesarean Section } \\
\hline No of cases & 16 & 22 & 10 & 4 & 14 & 10 \\
Gestation weeks & $25-30$ & $31-34$ & $35-39$ & $25-30$ & $31-34$ & $35-39$ \\
Respiratory Distress & 1 & 10 & 1 & 0 & 0 & 1 \\
Cerebral injury & 1 & 1 & 2 & 0 & 5 & 7 \\
Resp. Dist. +Cereb. inj. & 5 & 7 & 1 & 1 & 9 & 2 \\
Obitus & 9 & 0 & 0 & 3 & 0 & 0 \\
Nothing & 0 & 4 & 6 & 0 & 0 & 0 \\
\hline
\end{tabular}

The gestational ages at birth and reasons for hospitalization are shown above (Table). Forty-eight infants were born by spontaneous vaginal delivery and 28 by caesarean section. Twenty infants were born between 25 and 30 weeks gestation, 36 between 31 and 34 weeks and the remaining 20 between 35 and 39 weeks. Thirteen infants were admitted to the unit with respiratory distress syndrome, 16 with cerebral injury and a fur- 
ther 25 infants manifested a combination of these two conditions. Twelve infants manifested obitus and the remaining ten were normal healthy infants.

The annual twin birthrate breakdown was: $9.6 \%$ in $1990,15.4 \%$ in $1991,16.2 \%$ in 1992 and $6.4 \%$ in 1993 , representing $11.4 \%$ of the overall birthrate for those 4 years. Seventy-one per cent of the twin pairs were like-sexed; $28.9 \%$ FF and 42.1 MM. Of 661 newborns the mortality rate was $2.5 \%$ and for the twin infants the rate was $22.3 \%$. Eight twin infants $(10.5 \%)$ showed malformations at birth; the overall rate for malformations was $1.2 \%$.

As regards size at birth, $76.3 \%$ were appropriate for gestational age (AGA) while $23.7 \%$ were small for gestational age (SGA). Of those born before 31 weeks gestation $80 \%$ were females while after 31 weeks the majority were males $(66 \%)$. There was a within-pair growth discordancy $(>15 \%)$ in 13 of the 38 twin pairs.

The clinical histories of the mothers were also evaluated: $18.4 \%$ had undergone hormonal treatment (menopausal chorionic gonadotropin and/or progesterone); $31.5 \%$ had histories of hypertension, diabetes or hyperprolactinemia, and $5.7 \%$ of the pregnancies had resulted from assisted fecundation techniques. None reported previous twin pregnancies nor a family history of the phenomenon.

Data recorded are, evidentally, influenced by the reasons for admission to the intensive care unit and probably do not reflect the true incidence of disease. Accordingly, the incidence of morbidity ( 8 out of 76 infants) and mortality $22.3 \%$ is significantly high when compared to other reports. The incidence of caesarean delivery is also higher for twins than for singletons.

Respiratory distress syndrome (RDS) was more frequent in the spontaneous vaginal deliveries, whilst cerebral injury (CI) was more common in the caesarean deliveries and can be attributed to the diminished fetal adrenergic stimulation associated with this modality of birth. About $30 \%$ of the infants manifested RDS and CI combined. These however, were more clearly influenced by gestational age and size at birth than by delivery method.

The maternal histories of hormonal treatment, endocrine disease and assisted fecundation are factors also worth noting.

Despite the scantiness of our data, the study indicates the increased risk of morbidity and mortality for infants from multiple pregnancies and draws significant correlations between some infant characteristics (eg. gestational age and size at birth), delivery methods, maternal clinical history and the incidence of neonatal pathologies, such as, cerebral injury and respiratory distress syndrome.

\section{(Poster presentation)}

Correspondence: Dr. E. Gitto, Divisione Immaturi, Policlinico Universitario “Gazzi”, 98100 Messina, Italia. 